\title{
Optimizing Effectiveness in Electronic Prescriptions for Pediatric Outpatients: A Call for Responsive Action
}

\section{Richard H. Parrish II ${ }^{1, *}$, Sandra Benavides ${ }^{2}$, Joseph T. Malak ${ }^{3}$, Amy L. Potts ${ }^{4}$, Micheal Guirguis ${ }^{5}$ and Tracy Hagemann ${ }^{6}$}

1 Provincial Pharmacy Services, Alberta Health Services, Edmonton, AB T5H 3V9, Canada

2 Nova Southeastern University College of Pharmacy, Fort Lauderdale, FL 33328, USA; E-Mail: SBenavid@nova.edu

3 Department of Pediatrics at Vassar Brothers Medical Center, Poughkeepsie, NY 12601, USA; E-Mail: jmalak@bambini-peds.com

4 Department of Pharmacy at Monroe Carell Jr. Children's Hospital at Vanderbilt, Nashville, TN 37232, USA; E-Mail: amy.potts@vanderbilt.edu

5 Alberta Health Services and Faculty of Pharmacy and Pharmaceutical Sciences, University of Alberta, Edmonton, AB T6G 1Z1, Canada; E-Mail: Micheal.Guirguis@AlbertaHealthServices.ca

6 College of Pharmacy, University of Oklahoma, Oklahoma City, OK 73126, USA; E-Mail: Tracy-Hagemann@ouhsc.edu

* Author to whom correspondence should be addressed;

E-Mail: Richard.Parrish@AlbertaHealthServices.ca; Tel.: +1-780-735-6598; Fax: +1-780-735-4482.

Received: 15 August 2014; in revised form: 9 September 2014 / Accepted: 9 September 2014 / Published: 30 September 2014

\begin{abstract}
A pediatric compounded non-sterile products repository ( $\mathrm{pCNP}$ ) to optimize the effectiveness and safety of "off-label" use of compounded pharmacotherapy through complete transmission of electronic prescriptions across the continuum of care is described. The advent of electronic prescribing has the potential to refocus and resolve long-standing issues of prescription therapy for pediatric patients related to formulation, indication, dosing, and outcomes follow-up, among others. This white paper describes the architecture and function of the pCNP repository. Further, it calls on professional societies, health information technology (HIT) and pharmaceutical industries, universities, and government to create a safer pediatric pharmacotherapy system across the continuum of care. This system would include pCNPs within the existing federal and corporate database structures for medical language, and integrates advanced system safety features as requirements for prescribing, compounding, and dispensing non-mass produced prescription therapies for children.
\end{abstract}


Keywords: pediatrics; electronic prescriptions; compounded non-sterile products; repository

\section{Introduction}

The ability to provide evidenced-based effective and safe pharmacotherapy to pediatric patients has progressed tremendously in the past few decades. Historic acts of legislation such as the Pediatric Rule (1994), Food and Drug Administration Modernization Act (FDAMA) of 1997, Best Pharmaceuticals for Children's Act of 2002 (BPCA), and the Pediatric Research Equity Act of 2003 have emphasized the importance of clinical trials in this population. The cumulative effects have resulted in increased pharmacokinetic data, clinical trials, and increased consideration of pediatric drug formulations. However, there is still much room for improvement. Enormous strides have also been made with regard to the safe delivery of medication to children, including technology such as bar-coding, smart pumps, and computerized physician order entry (CPOE), with most of the focus being in the acute care setting. To date, minimal focus has been placed on the delivery of medications to pediatric patients outside of the acute or health-systems settings. In a health climate focused on improved outcomes and accountability, the time has come to enhance the medication use process in the community setting and across the continuum of care.

According to Surescripts, the largest electronic prescription intermediary in the world, over two-thirds of all physician offices in the US were actively utilizing electronic prescribing (or e-prescribing) in 2012 [1]. Moreover, in order to receive reimbursement for Medicaid patients, physician offices will be required to submit computerized records and e-prescriptions by 2015 under meaningful use provisions of the Accountable Care Act [2]. The American Academy of Pediatrics (AAP) recently endorsed e-prescribing for pediatric outpatients [3], and made a number of critical recommendations for improving electronic transmission from a provider's office or hospital to a community pharmacy. This list included indication-based dosing, common formulations for extemporaneously prepared dosage forms, pharmacies that will create extemporaneous compounds, and transmission of strength, concentration, and dose volumes, among others.

The practice of pediatrics has entered a time in which the need to address therapeutic, information-transfer and formulation issues related to prescription drug therapy for neonates, infants, children, and adolescents has never been more apparent or urgent. The advent of CPOE, in many ways magnifying the issues, has the potential to refocus and resolve the issues. Collaboration among the health professions, health information technology (HIT), pharmaceutical industries, universities, and government is necessary to create a better medication therapy system that meets the needs of all pediatric patients [4].

\section{Statement of the Problem}

A recent national estimate indicated at least 250 million prescriptions were dispensed at community pharmacies for approximately 30 million children in the U S. This is equivalent to an average of eight prescriptions annually per child [5]. Many of these prescriptions were initiated for an "off-label" use, that is, use not covered within the product's officially-recognized United States Food and Drug 
Administration (USFDA) labeling. Categories for off-label use include age, weight, contraindication, indication, and route of administration [6]. Prescriptions for dosage forms not identified within the labeling, such as compounded sterile products (CSPs) [7] and compounded non-sterile products (CNPs) [8], can be considered "off-label" uses. Often, the preparation of CSPs and CNPs is vital due to the lack of an available, suitable dosage formulation for the infant or child.

Moreover, the magnitude of e-transmitted prescriptions for CNPs dispensed for any patient, namely children, in any country on an annual basis is unknown. In addition, unknown is the extent to which computer software used in community pharmacies screen CNP prescriptions for potential medication therapy problems (e.g., drug allergy, drug-drug interactions, appropriate dose) as would occur for prescriptions with similar active ingredients dispensed in the manufacturer supplied dosage formulation. While many commercially-available oral antibiotic and analgesic liquids were among the top prescriptions dispensed for children [5], over 350,000 prescriptions for off-label use of lansoprazole alone were reported to be dispensed for patients less than 1 year old; most, if not all, of these prescriptions needed to be compounded. Inconsistent compounding practices could easily result in sub- or supratherapeutic dosing of any medication [9].

Evidence indicates that the generation of e-prescriptions may create a number of opportunities for medication errors and time delays in prescription processing [10,11]. Anecdotal evidence suggests that pediatricians and their office-based agents are required to hand-key all prescription-related information into a prescription transmission program without the benefit of dropdown menus for drug name, concentration, dosing and other important safeguards. Moreover, the new morbidities of the twenty first century in pediatric patients, such as the dramatic rise in chronic conditions like obesity, attention-deficit hyperactivity disorder (ADHD), anxiety, depression, bipolar disorder (BPD), and Asperger's Syndrome are being applied to increasingly younger children. These uncharted waters further heighten uncertainty regarding $\mathrm{pCNP}$ prescription and formulation.

Medicines information databases are often populated into EMR and CPOE for readily accessible dosing information. The medicines database, however, may not be pediatric specific. Additionally, the information related to CNPs in not readily available in most of these commercially available databases. Pharmacists receiving such prescriptions in the community are tasked with verifying the appropriate indication, dose, and formulation to prepare for the patient. The pharmacist also needs the necessary scientific-based information on the appropriate recipe for preparation. These gaps may discourage pharmacists in the community from dispensing such medications for children due to the lack of available information, the lack of reimbursement from insurance companies, and inexperience with such products. This may result in non-adherence to the medication and less than optimal patient outcomes.

A standardized data set for extemporaneous pCNPs is not part of the National Library of Medicine Unified Medical Language System's (UMLS) RxNorm and RxTerms. The UMLS serves to normalize drug name mediators to allow communication between pharmacy management and drug interaction software [12]. Like companion corporate and commercial counterparts, this database includes only mass-produced, commercially-available products designated by a National Drug Code (NDC), a Uniform Product Code (UPC), or similarly encoded product identifiers, such as Veterans Administration National Drug File. However, when a pharmacist compounds a prescription for an individual patient, that workproduct becomes commercially-available. Methodology to codify and include this type of evidencebased, peer-reviewed work-product in standardized product listings should be developed. 
Clearly, the problems of pediatric pharmacotherapeutics and medication management are not unique to any particular country. Since 2007, the World Health Organization has emphasized the unique nature of medication selection and use in children by creating the Model List for Essential Medicines for Children [13]. The Dutch Pediatric Formulary, an electronic database, includes information on standardized pCNP concentrations, as well as indications-based dosing, contraindications, and precautions [14]. In one North Carolina health system, efforts to standardize pCNP formulas via a centralized computerized database have been underway to establish evidence-based beyond-use dates (BUD), reduce the potential for medication errors related to nomenclature mismatches and formulation, and improve patient safety relative to the process for prescription generation and transmission [15]. Recently, a Michigan consortia consisting of professional societies, university, and healthcare organizations created a website to improve patient safety through standardized oral liquid concentrations [16]. While the number of new molecular entities with either pediatric nosology or posology in official product labeling has increased exponentially since enactment of BPCA [17], scientific evidence for the use, preparation, and monitoring of currently marketed drugs remains elusive [18-20].

Thus, there is a tremendous need to both compile and assign appropriate identifiers, dosage form designations, and medicines use information for pCNPs to facilitate e-prescription transmission and dispensing for children. There is a parallel need for the professions to take ownership for the creation of a repository that would assemble, maintain, and expand computerized listings for all medications that have peer-reviewed scientific literature supporting pediatric formulation and dosing. The purpose of this white paper is to outline the framework and architecture for this repository to facilitate the electronic identification, standardization, and transmission of CNPs for pediatric patients irrespective of the location of e-prescription generation.

\subsection{Flow Impedance for $p$ CNP E-Prescriptions in the Current Medication Management System}

The current processes for generating and dispensing CNP prescriptions for children in both in-patient and out-patient environments have a number of impedances or disruptions, often necessitating enactment of alternate processes called "workarounds." [21] Workarounds are associated with a number of individual and systematic factors related to work design, responses to problems, and consequences that perpetuate the generation of future errors. In short, workarounds result in a greater probability for error because they increase process variation among practitioners.

In a typical scenario for the generation of an e-prescription for a CNP, a number of impedances or workarounds are required for order fulfillment across the continuum of care. After the provider assesses the need for a medication, the prescription is manually typed into an available text-entry field, often without the benefit of dropdown menus for product selection, dosing unique to the medical indication, dose volume or mass descriptors, or total quantity needed. Once this information is entered, the information is routed to the electronic inbox of a community pharmacy that may or may not be able to prepare any particular pCNP. Instead of completing receipt of the prescription by importing the information into compatible prescription information processing fields, the community pharmacy needs to append the transmission, and create a new manually-entered prescription, again without the benefit of pre-formatted dropdown menus for correct spelling, concentration, and dose volume. If the prescription information seems complete or if ambiguities are not identified, the pCNP is prepared with or without peer-reviewed 
scientific formulation information. These prescriptions are not filtered through electronic screening to identify potential drug therapy problems because their entries are not coded to match existing databases.

The ultimate impedance is that the patient does not have the prescription dispensed because of processing delays, lack of expertise to formulate the product, or lack of access to an active pharmaceutical ingredient (API) that is accessible to an adult patient. At and within each disruption, multiple opportunities for provider, compounder, and technical error are present, further expanding the risk for patient harm. Healthcare processes have multiple steps within the medication use system. Without standardization, these processes become error prone. An equally error-prone medication use process within a health-care system would not be acceptable to accreditation and regulatory agencies, such as the Joint Commission.

Moreover, in pediatric acute care settings that utilize CPOE, pCNP drug formulary databases are often built from a hybrid list of institution-specific and evidence-based formulae, and do not allow a bidirectional flow of complete and accurate prescription information between service providers [22]. This lack of interoperability creates a number of opportunities for error during transitions of care from one setting to another [23], especially during hours when either pharmacy is closed. Research has documented challenges with medication reconciliation related commonly to dose and frequency accuracy for admitted in-patients; however, e-transmission of pCNP prescriptions for these patients at discharge is often impeded due to lack of a shared drug nomenclature and coding system designed to identify the API and its strength [22,24]. In short, there is a vital need to create a durable "source of truth" regarding pCNPs in an electronic medium so that prescription records are completely transferrable between levels of care and contain standardized and evidence-based formulations.

\section{2. pCNP Repository Design Considerations}

To address the impedance factors illustrated above, an evidence-based pCNP repository is proposed to have six sub-directories: (1) a standardized name with concentration or listing descriptors; (2) a standardized numerical code based on the molecule's generic name; (3) the excipient descriptors; (4) peer-reviewed scientific literature citations for dosing or formulation; (5) unique indications-based dosing information; and (6) safety and effectiveness coding (see Figure 1). Each of these sub-directories would be written in such a way to be compatible with HL7 language [25]. The repository would be maintained and expanded by an appropriate professional organization or a consortium of organizations devoted to safe medication use in pediatrics. Its Research and Development (R\&D) arm would be responsible for identifying gaps in product listings. The identified gaps will create a research agenda for scientific work, and would serve as an impetus to solicit funding to support the generation of research publications to populate and substantiate the repository. Additionally, research supported through residencies and fellowships and partnerships with small business would also contribute to the repository. Similar to the European Pediatric Formulation Initiative [26], a professional organization or consortium would initiate the creation of the pCNP repository, establish business relationships with related software vendors, foster advocacy among manufacturers for commercially-available formulation, and create the business plan for its activation. In the absence of such effort, repository creation redounds to government to address patient safety and commercial issues. Regardless of its owner, the repository would be a "living" organ built to aid evidence-based use and preparation of medications. 
Figure 1. Pediatric compounded non-sterile product e-prescription repository (pCNP) architecture.

\begin{tabular}{|c|c|c|c|c|c|}
\hline $\begin{array}{l}\text { Standardized } \\
\text { product } \\
\text { descriptions, } \\
\text { including } \\
\text { name and } \\
\text { standardized } \\
\text { concentration }\end{array}$ & $\begin{array}{l}\text { Standardized } \\
\text { generic-name } \\
\text { numerical } \\
\text { Clinical } \\
\text { Formulation } \\
\text { ID (CF-ID) } \\
\text { code }\end{array}$ & $\begin{array}{l}\text { Excipient } \\
\text { descriptors } \\
\text { linked to } \\
\text { RxNorm }\end{array}$ & $\begin{array}{c}\text { Peer- } \\
\text { reviewed } \\
\text { scientific } \\
\text { literature / } \\
\text { PA / REMS } \\
\text { mechanisms }\end{array}$ & $\begin{array}{l}\text { SNOMED } \\
\text { indications- } \\
\text { based dosing } \\
\text { codes }\end{array}$ & $\begin{array}{l}\text { MEDWATCH } \\
\text { or ISMP } \\
\text { safety and } \\
\text { effectiveness } \\
\text { codes }\end{array}$ \\
\hline
\end{tabular}

About 250 APIs have published scientific formulation and dosing information [27-29] and can easily serve as the initial agents in the pCNP repository. The indications-based dosing information sub-directory can begin with oncologic-related preparations formulated within the protocols from the Children's Oncology Group (COG) [30]. Many child-friendly excipients used in pCNP prescription preparation, such as suspending and flavoring agents, are currently listed in available commercial and governmental repositories. Based on published peer-reviewed stability data, the agents could be linked to the base molecule listed in the standardized product description. The next sequence would link the complete medication order with literature citations to support any required pre- or prior authorization requirements or to activate a Risk Evaluation and Mitigation Strategy (REMS) [31], informed consent, or other required medication information when appropriate. In the fourth step, once the indication is selected, dosing specific to that particular use, if applicable, would be linked using Systematized Nomenclature of Medicine (SNOMED) language [32]. In the final step, the repository would house any relevant patient outcomes by linking a unique transaction number with FDA's MedWatch [33] or Institute for Safe Medication Practices (ISMP) [34] safety and effectiveness codes. This last step would require additional coordination among providers to determine authorized persons for assessment, collection, and entry of these data.

Because a standardized medication informatics, preparation and use system for children would identify and mitigate potential patient risks related to formulation and dosing, opportunities to improve both the safety and effectiveness of pharmacotherapy would be optimized. The ability to incorporate drugs in Phase II and III clinical testing would serve an efficient research and development feedback not currently possible in either community or hospital practice. A full description of this system aspect for a research purpose is beyond the scope of this paper. Suffice it to say that all clinical testing in children, including research for off-label use, could be accommodated within this system as a vehicle for modifying product labeling, building the scientific infrastructure for practice-based research in pediatric pharmacotherapy, harmonizing pCNP formulations between patient care environments (institutional and community), and improving both the quality and quantity of knowledge translation and dissemination. 


\section{Conclusion and Call to Responsive Action}

The Affordable Care Act will have a major impact on the access to care for pediatric patients and the practice and medication use in children. In addition, there are many unknowns with regard to the programmatic operations and patient outcomes. A major unknown is how the formularies associated with the affordable health plans, applied to dramatically larger segments of the population, will serve the needs of children, since pCNPs typically are not covered under many current third party insurance plans, including Medicaid. The creation of a pCNP electronic repository has the potential to increase children's access to medically necessary pharmacotherapies. It also has the ability to improve transitions of care for patients as more community pharmacists will have access to the information needed to prepare the medication in the same formulation obtained in the acute care setting.

The health professions have an important responsibility for fulfilling access to safe, effective, and evidence-based formulations, including pCNPs, as part of the social contract with children. In the absence of responsiveness by professional, university, or corporate entities, government has a basic welfare obligation to the children of the United States to coordinate the enhancement of existing federal systems, like UMLS and its constituent databases, to remedy these informational deficiencies by including evidence-based pCNP formulations. Otherwise, treatment of neonates, infants, children, and adolescents will continue to fall short of optimal and coordinated medication use. In the wake of the New England Compounding Center (NECC) debacle and in the era of pediatric accountable care, need the professions and industry wait for the next calamity before we act responsively?

\section{Declaration}

The views expressed in this paper are those of its authors, and do not necessarily represent official policy or positions by the authors' respective institutions or organizations.

The Board of Directors of the Pediatric Pharmacy Advocacy Group endorses this paper.

\section{Acknowledgements}

The authors gratefully acknowledge the contributions and suggestions of members of the Pediatric Pharmacy PBRN on LinkedIn [35].

\section{Author Contributions}

Richard Parrish conceived the original repository idea and prepared the initial manuscript. Sandra Benavides wrote the introduction and coordinated manuscript editing. Joseph Malak and Amy Potts reviewed the manuscript and provided examples from actual practice. Micheal Guirguis reviewed the manuscript and wrote the abstract. Tracy Hagemann reviewed the manuscript and made substantive recommendations on repository structure.

\section{Conflicts of Interest}

The authors declare no conflict of interest. 


\section{References}

1. The National Progress Report for Eprescribing Year 2012. Available online: http://www.surescripts.com/about-e-prescribing/progress-reports/national-progress-reports.aspx (accessed on 20 July 2013).

2. Meaningful Use. Centers for Medicare and Medicaid Services. Department of Health and Human Services. Available online: http:/www.cms.gov/Regulations-and-Guidance/Legislation/ EHRIncentivePrograms/Meaningful_Use.html (accessed on 24 September 2013).

3. Johnson, K.B.; Lehmann, C.U. Council on Clinical Information Technology. Electronic prescribing in pediatrics: Toward safer and more effective medication management. Pediatrics 2013, 131, e1350-e1356.

4. Bhatt-Mehta, V.; Buck, M.L.; Chung, A.; Farrington, E.A.; Hagemann, T.M.; Hoff, D.S.; LaRochelle, J.M.; Pettit, R.S.; Phan, H.; Potts, A.L.; et al. Recommendations for meeting the pediatric patient's need for a clinical pharmacist: A Joint Opinion of the American College of Clinical Pharmacy Pediatrics Practice and Research Network and the Pediatric Pharmacy Advocacy Group. Pharmacotherapy 2013, 33, 243-251.

5. Chai, G.; Governale, L.; McMahon, A.W.; Trinidad, J.P.; Staffa, J.; Murphy, D. Trends of outpatient prescription drug utilization in US children, 2002-2010. Pediatrics 2012, 130, 23-31.

6. Kimland, E.; Odlind, V. Off-label drug use in paediatric patients. Clin. Pharmacol. Ther. 2012, 91, 796-801.

7. USP37-NF32. Chapter <797> Pharmaceutical Compounding-Sterile Preparations; The United States Pharmacopeial Convention, Inc.: Rockville, MD, USA, 2014.

8. USP37-NF32. Chapter <795> Pharmaceutical Compounding-Non-Sterile Preparations; The United States Pharmacopeial Convention, Inc.: Rockville, MD, USA, 2014.

9. Noel, G.J.; van den Anker, J.N.; Lombardi, D.; Ward, R. Improving drug formulations for neonates: Making a big difference in our smallest patients. J. Pediatr. 2010, 161, 947-949.

10. Palchuk, M.B.; Fang, E.A.; Cygielnik, J.M.; Labreche, M.; Shubina, M.; Ramelson, H.Z.; Hamann, C.; Broverman, C.; Einbinder, J.S.; Turchin, A. An unintended consequence of electronic prescriptions: Prevalence and impact of internal discrepancies. J. Am. Med. Inform. Assoc. 2010, 17, 472-476.

11. Lapane, K.L.; Rosen, R.K.; Dubé, C. Perceptions of eprescribing efficiencies and inefficiencies in ambulatory care. Int. J. Med. Inform. 2011, 80, 39-46.

12. National Library of Medicine. Unified Medical Language System, RxNorm. Available online: http://www.nlm.nih.gov/research/umls/rxnorm/ (accessed on 20 July 2013).

13. World Health Organization Model List of Essential Medicines for Children. Available online: http://www.who.int/medicines/publications/essentialmedicines/en/ (accessed on 5 September 2013).

14. Nederlands Kenniscentrum Farmacotherapie bij Kinderen, Kinderformularium. Available online: http://www.kinderformularium.nl (accessed on 20 July 2013).

15. Haile, M.; Anderson, K.; Evans, A.; Crawford, A. Development of a standardized Intranet database of formulation records for nonsterile compounding, Part 2. Int. J. Pharm. Compd. 2012, 16, 104-109. 
16. Michigan Pediatric Safety Collaboration. State-wide initiative to Standardize the Compounding of Oral Liquids in Pediatrics. Available online: http://www.mipedscompounds.org (accessed on 12 February 2014).

17. National Institute of Child Health and Human Development. Best Pharmaceuticals for Children. Available online: http://bpca.nichd.nih.gov/ (accessed on 11 September 2013).

18. Sachs, A.N.; Avant, D.; Lee, C.S.; Rodriguez, W.; Murphy, M.D. Pediatric information in drug product labeling [research letter]. JAMA 2012, 307, 1914-1915.

19. Shelton, C.; Chhim, R.F.; Christensen, M.L. Recent new drug approvals. Part 1: Drugs with pediatric indications. J. Pediatr. Pharmacol. Ther. 2012, 17, 329-339.

20. Chhim, R.F.; Shelton, C.; Christensen, M.L. Recent new drug approvals. Part 2: Drugs undergoing active clinical studies in children. J. Pediatr. Pharmacol. Ther. 2013, 18, 14-38.

21. Spear, S.J.; Schmidhofer, M. Ambiguity and workarounds as contributors to medical error. Ann. Intern. Med. 2005, 142, 627-630.

22. Porcelli, P.J.; Waltman, L.R.; Brown, S.H. A review of medication reconciliation issues and experiences with clinical staff and information systems. Appl. Clin. Inform. 2010, 1, 442-461.

23. Huynh, C.; Wong, I.C.; Tomlin, S.; Terry, D.; Wilson, K.; Jani, Y. Medication discrepancies at transitions in pediatrics: A review of the literature. Pediatr. Drugs. 2013, 15, 203-215.

24. Dersch-Mills, D.; Hugel, K.; Nystrom, M. Completeness of information sources used to prepare best possible medication histories for pediatric patients. Can. J. Hosp. Pharm. 2011, 64, 10-15.

25. Health Level Seven International. Available online: http://www.hl7.org/ (accessed on 11 September 2013).

26. European Paediatric Formulations Initiative. STEP database-Safety and toxicity of excipients in paediatrics. Available online: http://www.eupfi.org/index.html (accessed on 22 September 2013).

27. Nahata, M.C.; Pai, V.B. Pediatric Drug Formulations, 6th ed.; Harvey Whitney Books: Cincinnati, $\mathrm{OH}, \mathrm{USA}, 2011$.

28. Nahata, M.C. Lack of pediatric dosage formulations. Pediatrics 1999, 104, 607-609.

29. Strickley, R.G.; Iwata, Q.; Wu, S.; Dahl, T.C. Pediatric drugs-A review of commercially available oral formulations. J. Pharm. Sci. 2008, 97, 1731-1774.

30. Children's Oncology Group. The World's Childhood Cancer Experts. Available online: http://childrensoncologygroup.org/ (accessed on 15 September 2013).

31. National Library of Medicine. SNOMED (Systematized Nomenclature of Medicine) Clinical Terms. Available online: http://www.nlm.nih.gov/research/umls/Snomed/snomed_main.html (accessed on 11 September 2013).

32. Department of Health and Human Services. United States Food and Drug Administration. Approved Risk Evaluation and Mitigation Strategies (REMS). Available online: http://www.fda.gov/drugs/drugsafety/postmarketdrugsafetyinformationforpatientsandproviders/uc m111350.htm (accessed on 11 September 2013).

33. United States Food and Drug Administration. MedWatch: The FDA Safety Information and Adverse Event Reporting Program. Available online: http://www.fda.gov/safety/medwatch/ default.htm (accessed on 15 September 2013). 
34. Institute for Safe Medication Practices. A Nonprofit Organization Educating the Healthcare Community and Consumers about Safe Medication Practices. Available online: http://www.ismp.org (accessed on 15 September 2013).

35. Pediatric Pharmacy PBRN. Available online: http://www.linkedin.com/groups/PediatricPharmacy-PBRN-4621858?trk=my_groups-b-grp-v (accessed on 9 September 2014)

(C) 2014 by the authors; licensee MDPI, Basel, Switzerland. This article is an open access article distributed under the terms and conditions of the Creative Commons Attribution license (http://creativecommons.org/licenses/by/4.0/). 